\title{
Co/Ni multilayers for spintronics: High spin polarization and tunable magnetic anisotropy
}

\author{
S. Andrieu, ${ }^{1, *}$ T. Hauet, ${ }^{1}$ M. Gottwald,${ }^{1, \dagger}$ A. Rajanikanth, ${ }^{1, \dagger}$ L. Calmels,${ }^{2}$ A. M. Bataille, ${ }^{3}$ F. Montaigne,${ }^{1}$ S. Mangin, ${ }^{1}$ \\ E. Otero, ${ }^{4}$ P. Ohresser, ${ }^{4}$ P. Le Fèvre, ${ }^{4}$ F. Bertran, ${ }^{4}$ A. Resta, ${ }^{4}$ A. Vlad, ${ }^{4}$ A. Coati, ${ }^{4}$ and Y. Garreau ${ }^{4,5}$ \\ ${ }^{1}$ Institut Jean Lamour, UMR CNRS 7198, Université de Lorraine, 54000 Nancy \\ ${ }^{2}$ CEMES-CNRS, Université de Toulouse, BP 94347, F-31055 Toulouse Cedex 4, France \\ ${ }^{3}$ Laboratoire Léon Brillouin, IRAMIS, CEA Saclay, 91191 Gif sur Yvette, France \\ ${ }^{4}$ Synchrotron SOLEIL-CNRS, L'Orme des Merisiers, Saint-Aubin, BP48, 91192 Gif-sur-Yvette, France \\ ${ }^{5}$ Laboratoire Matériaux et Phénomènes Quantiques, Université Paris Diderot, 75013 Paris, France
}

(Received 7 February 2018; revised manuscript received 17 April 2018; published 27 June 2018)

\begin{abstract}
In this paper we analyze, in detail, the magnetic properties of $(\mathrm{Co} / \mathrm{Ni})$ multilayers, a widely used system for spintronics devices. We use spin-polarized photoemission spectroscopy, magneto-optical Kerr effect, x-ray magnetic circular dichroism (XMCD), and anomalous surface diffraction experiments to investigate the electronic, magnetic, and structural properties in $[\mathrm{Co}(x) / \mathrm{Ni}(y)]$ single-crystalline stacks grown by molecular-beam epitaxy. The spin polarization depends sensitively on the surface termination and for Co terminated stacks is found to be much larger than bulk Co, reaching at least $90 \%$ for 2 Co atomic planes. We observe a magnetization transition from in plane to out of plane when varying the Ni coverage on a Co layer in the submonolayer range, confirming the interface origin of the perpendicular magnetic anisotropy in this system. Angle-dependent XMCD using strong applied magnetic field allows us to show that the orbital magnetic moment anisotropy in Co is responsible for the anisotropy and that our results are consistent with Bruno's model. Surface x-ray diffraction shows that fcc stacking is preferred for 1-monolayer Co-based superlattices, whereas the hcp stacking dominates for larger Co thicknesses. We finally explored the role of the stacking sequence on the Co and Ni magnetic moments by $a b$ initio calculations.
\end{abstract}

DOI: 10.1103/PhysRevMaterials.2.064410

\section{INTRODUCTION}

Research on spintronic devices such as magnetic random access memories (MRAM) and magnetic sensors have generated the need for magnetic thin-film materials with tunable properties. Although today most spintronic devices are based on a similar spin-valve magnetic stack for sensing, writing, and reading operations, each application requires specific optimized features for the magnetic films. For instance, in the view of spin transfer torque (STT) MRAM [1,2] and STT oscillators $[1,3]$ implementation, magnetic tunnel junction (MTJ) with thin electrodes having low damping, high perpendicular anisotropy, high spin polarization, and moderate magnetization are investigated. Different ways are heavily pursued. One involves rare-earth/transition metal ferrimagnet alloys [4]. Another one is $\left[\mathrm{Fe}_{1-\mathrm{x}} \mathrm{Co}_{\mathrm{x}} / \mathrm{Pd}\right]$ and $\left[\mathrm{Fe}_{1-\mathrm{x}} \mathrm{Co}_{\mathrm{x}} / \mathrm{Pt}\right]$ multilayers [5-7]. Both show large perpendicular magnetocrystalline anisotropy (PMA) and allow easy tuning of magnetization. However these systems offer only modest spin-polarization and high damping parameters [8]. On the contrary Heusler alloys are heavily studied since they are theoretically $100 \%$ spin polarized with extremely low damping

\footnotetext{
*Corresponding author: stephane.andrieu@univ-lorraine.fr

${ }^{\dagger}$ Present address: IBM - T. J. Watson Research Center, Yorktown Heights, New York 10598, USA.

${ }^{\ddagger}$ Present address: University of Hyderabad, Hyderabad 500046, Telangana, India.
}

$(\alpha<0.001)$. A high tunnel magnetoresistance (TMR) ratio has indeed been reported for magnetic tunnel junctions with Co-based full Heusler alloy electrodes [9-11] and damping below 0.001 was reported [12,13] but large perpendicular magnetic anisotropy (PMA) is still challenging in this class of materials.

$[\mathrm{Co} / \mathrm{Ni}]$ multilayers have gained large interest in view of spin-transfer applications since it has the potential to provide all the requested features cited above $[14,15]$. First, the $\mathrm{Co} / \mathrm{Ni}(111)$ system is ideal regarding growth processes: as both $\mathrm{Ni}$ and Co grows layer by layer on each other (as shown by electron-diffraction intensity oscillations [15]), extremely flat interfaces are obtained as shown by transmission electron microscopy [15]. The $\mathrm{Co} / \mathrm{Ni}$ interface produces a magnetic anisotropy that is perpendicular to the interface [16-18]. PMA up to $5 \mathrm{MJ} / \mathrm{m}^{3}$ can be achieved for superlattices of 1 MonoLayer (ML) of Co and 3 MLs of Ni grown along the (111) direction $[5,15,19]$. Changing the thickness of Co allows an easy tuning of PMA amplitude and calculations lead to similar conclusions [20-22]. Magnetization of $\mathrm{Co} / \mathrm{Ni}$ multilayers is moderate (around $700 \mathrm{emu} / \mathrm{cm}^{3}$ for Co $1 \mathrm{ML} / \mathrm{Ni} 3 \mathrm{MLs}$ ). Gilbert damping has been found to be quite insensitive to the composition and can reach values around 0.02 [23-25]. The importance of such a set of characteristics to enhance spin transfer has been recently demonstrated. First reliable STT switching has been achieved in fully metallic $\mathrm{Co} / \mathrm{Ni}$-based GMR nanopillars for low critical current [26,27] and for subnanosecond time [28]. Second recent reports on STT-induced 
domain-wall motion have demonstrated low critical current [29] and high domain-wall speed in $\mathrm{Co} / \mathrm{Ni}$ [30-32]. Finally, a high spin polarization (denoted as SP in the following) was predicted using $a b$ initio calculation [33] and indirectly estimated experimentally [30]. The main problem to use $\mathrm{Co} / \mathrm{Ni}$ in magnetic tunnel junction is to find a suitable insulating barrier for getting large tunnel magnetoresistance [14]. Encouraging results were recently reported using an $\mathrm{Al}_{2} \mathrm{O}_{3}$ barrier [34].

However while $\mathrm{Co} / \mathrm{Ni}$ multilayers have been extensively studied (see [15] for a review and [19,25,31-35]) many fundamental questions about this system have still to be addressed. These include the following:

(i) Spin polarization. We performed spin-resolved photoemission (SR-PES) experiments to quantify the SP dependence on $\mathrm{Co}$ and $\mathrm{Ni}$ layer thicknesses and show that SP of at least $90 \%$ can be achieved for 2-ML Co on Ni (Sec. II).

(ii) Interfacial anisotropy contributions. It is well established that interfacial anisotropy gives rise to PMA values sufficient to overcome the shape anisotropies. In all the multilayers studied in the literature, Co is always embedded in $\mathrm{Ni}$, leading to two $\mathrm{Co} / \mathrm{Ni}$ interfaces and so two interfacial contributions. But if we need to finish the stack with Co in transport devices, a Ni/Co interface is lacking and the PMA will be modified. We thus studied the Ni surface coverage on Co to quantitatively determine the different interfacial magnetic contributions to the PMA (Sec. III A).

(iii) Atomic magnetic moments. In a previous study we observed that the Co magnetic moment (measured at room temperature) is enhanced at the $\mathrm{Ni}$ interface [15]. Here we performed x-ray magnetic circular dichroism (XMCD) measurements in a special geometry that allows us first to quantify the true Co and Ni spin moments (by determining and correcting the artefact due to the spin magnetic dipole operator at the interface) and second to measure the anisotropy of the orbital moment at the atomic level. Finally, our data can be used to test if Bruno's PMA explanation [36] correctly describes our observations (Sec. III B).

(iv) Discrepancies between experimental and theoretical magnetic properties. In our previous study we performed $a b$ initio calculations to understand in detail the mechanism that leads to PMA in this system. We did not find theoretically that the Co moment is enhanced at the interface like in experiments. One explanation could be a special Co atomic arrangement at the interface with Ni not explored when performing ab initio calculations. To address this point, we performed anomalous $\mathrm{X}$-ray surface diffraction experiments to determine the atomic arrangement in the system (Sec. IV). We performed ab initio calculations looking at the influence of the Co stacking sequence (fcc versus hcp staking) on the hexagonal (111) fcc Ni lattice (Sec. V).

Finally all these results are summarized and discussed (Sec. VI), where we propose a simple model to account for both the high Co moments and almost full spin polarization.

\section{Co/Ni SPIN POLARIZATION}

In order to investigate the $\mathrm{SP}$ of $[\mathrm{Co} / \mathrm{Ni}]$ superlattices, spin-resolved photoemission spectroscopy (SR-PES) experi- ments were performed on the CASSIOPEE beamline at the SOLEIL synchrotron (see Ref. [37] for a description of the experimental setup). The $\mathrm{Co} / \mathrm{Ni}$ stacks were epitaxially grown in a MBE chamber connected to the beamline to keep the surface contamination to an insignificant level [12]. We used the same growth process as developed in Refs. [15,18]. The films were deposited on (1120) single-crystalline sapphire substrates. To deposit $\mathrm{Co} / \mathrm{Ni}$ superlattices with (111) growth direction, seed layers of bcc V (110) $10 \mathrm{~nm} / \mathrm{fcc} \mathrm{Au}$ (111) $5 \mathrm{~nm}$ were first deposited. A series of $[\mathrm{Ni}(3 \mathrm{ML}) / \mathrm{Co}(x)]_{3}$ superlattices were measured with $x$ ranging from 1 to 3 monolayers (MLs) with 0.5-ML step. 20-nm-thick (0001) hcp Co and (111) fcc Ni films were also grown as bulk references. All $\mathrm{Co} / \mathrm{Ni}$ superlattices have been magnetized with a 600-Oe field applied perpendicularly to the films before PES experiments (and in-plane for thick $\mathrm{Ni}$ and Co reference films). Preliminary experiments have shown that such a magnetic field is sufficient to saturate the $\mathrm{Co} / \mathrm{Ni}$ magnetization [18]. The SR-PES experiments have been conducted at room temperature with a spectrometer detector facing the sample surface with an angular integration to $+/-8^{\circ}$. The spectrometer is equipped with a Mott detector allowing us to measure the SP along the in-plane and out-of-plane directions and thus allowing us to check the magnetic anisotropy features of the $\mathrm{Co} / \mathrm{Ni}$ stacks. Most of the experiments were performed using a 37-eV photon energy where the photoemission cross section is the largest for Co and Ni. Such conditions probe around $40 \%$ of the first Brillouin zone (BZ) in the $k_{x}-k_{y}$ plane. To investigate the whole BZ, we thus turned the sample normal $8^{\circ}$ off the detector axis. The PES experiments were performed on a $(\mathrm{Co} x \mathrm{ML} / \mathrm{Ni}$ $3 \mathrm{ML}) * N$ superlattice series. The Ni thickness was fixed to $y=3$ MLs (which is not a critical parameter since the PMA does not depend on it in our samples [15]). The Co thickness was varied from $x=1-3$ MLs by 0.5 -ML steps. The number of repetitions $N$ was fixed to 3 first to be sure to get the PMA and second because the electron escape depth $l_{\text {esc }}$ is around 2 MLs so that the PES probing depth is around $1 \mathrm{~nm}$ below the surface in our experimental conditions (95\% of PES signal comes from $3 . l_{\mathrm{esc}}$ ). In Fig. 1 the majority and minority SR-PES and resulting SP for this series are plotted, including thick $\mathrm{Ni}$ and Co films (for which the magnetization was observed in plane).

The SP at the Fermi energy is much larger than in bulk Ni and Co and reaches $90 \%$ around 2-ML Co. The SP increase is attributed to an increase of the minority spin PES near $E_{F}$ whereas the majority spin PES is weakly affected as indicated by the arrows in Fig. 1.

We also looked at the impact of nonmagnetic capping on the Co layer on the SP. In Fig. 2 is shown the effect of Ni and Au covering on the SP at the surface. As expected the high SP obtained by a Co termination is reduced when covering with $\mathrm{Ni}$. This leads to the conclusion that the use of Co/Ni SL as an electrode should be terminated with Co. The Au capping was also examined and the initial SP is slightly decreased. In practice the SP of the underneath Co layer is nearly unaffected because there is some unpolarized Density Of States (DOS) coming from $\mathrm{Au}$ that is included in the calculation of the SP. This means that a very high spin-polarized current can be injected in an $\mathrm{Au}$ spacer using a $\mathrm{Co} / \mathrm{Ni} \mathrm{SL}$ terminated with Co. 

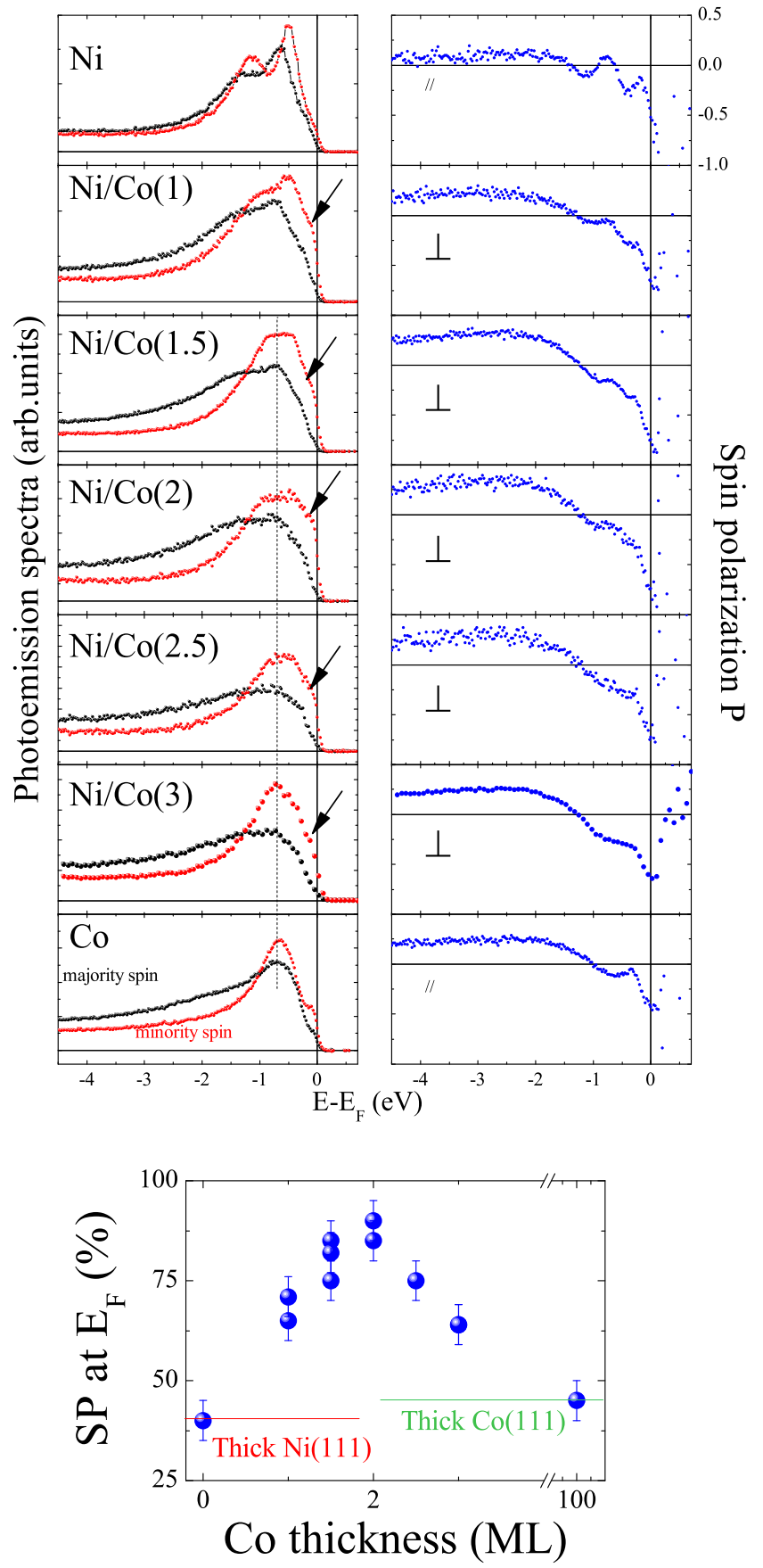

FIG. 1. Top left: majority and minority spin PES spectra measured on bulk $\mathrm{Ni}$ and $\mathrm{Co}$ films and a series of $\mathrm{Co} / \mathrm{Ni}$ superlattices. Top right: spin polarization spectra; bottom: corresponding spin polarization (SP) at $E_{F}$. The arrows show the increase of the minority spin PES near $E_{F}$ responsible for the SP increase.

\section{MAGNETIC ANISOTROPY}

\section{A. MOKE investigation}

To inject a high polarized spin current in a $\mathrm{Co} / \mathrm{Ni}$ based device, the superlattice must be terminated by a Co layer. But this leads to the lack of $1 \mathrm{Co} / \mathrm{Ni}$ interface that can be enough to lose the PMA. To test this point, stacks were grown with one $\mathrm{Ni} / \mathrm{Co}$ first interface with a varying $\mathrm{Ni}$ capping coverage
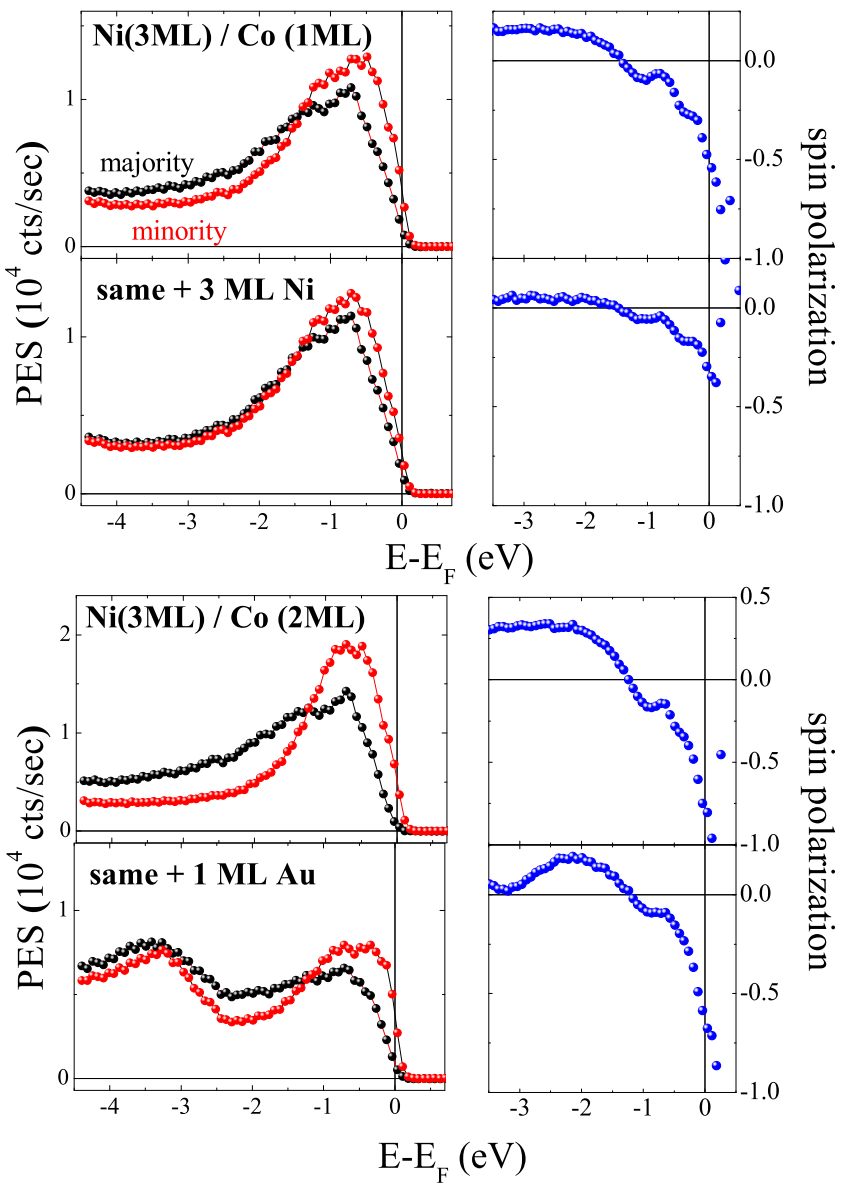

FIG. 2. Effect of the covering on the spin polarization of the stack for 3-ML Ni capping (top) and for 1-ML Au capping (bottom). This shows that Ni covering decreases the SP whereas an almost full polarized injection may be achieved in Au.

as shown in Fig. 3(a). The whole sample was then capped with $\mathrm{MgO}$. The architecture of the sample is thus sapphire $/ \mathrm{V}(110) / \mathrm{Au}(111) / \mathrm{Ni}_{3 \mathrm{ML}} / \mathrm{Co}_{\mathrm{xML}} / \mathrm{Ni}_{\mathrm{yML}} / \mathrm{MgO}$ where the top $\mathrm{Ni}$ layer is a thickness wedge with $0 \leqslant y \leqslant 1.2$ MLs. Details on the growth are given previously [15]. One should note that Co growth on $\mathrm{Ni}$ and $\mathrm{Ni}$ growth on $\mathrm{Co}$ is layer by layer in these samples as shown by electron diffraction (reflection high-energy electron diffraction oscillations). This means that a submonolayer $\mathrm{Ni}$ thickness consists of two-dimensionnal islands and a noncoalesced monolayer. Thus the top $\mathrm{Co} / \mathrm{Ni}$ interface proportion is equal to $y$ (for $y \leqslant 1$ ). The hysteresis loops were measured along the Ni wedge by using magnetooptical Kerr effect (MOKE) microscopy applying the field perpendicular to the layers [Fig. 3(b)]. As expected for a 3ML-thick Co layer without Ni coverage, the out-of-plane axis was found to be a hard axis. Indeed, a single Co-Ni interface anisotropy is not sufficient to overcome the demagnetization field. This is still the case up to a $0.5-\mathrm{ML} \mathrm{Ni}$ cap, but the anisotropy (the loop slope) changed around 0.5-ML Ni cap and the out-of-plane axis became an easy axis for $\mathrm{Ni}$ cap thicknesses above 0.7 ML. Similar experiments were done for Co thickness varying from 1 to 4 MLs. We deduced from these experiments the Ni cap thickness $y$ for which the magnetization 

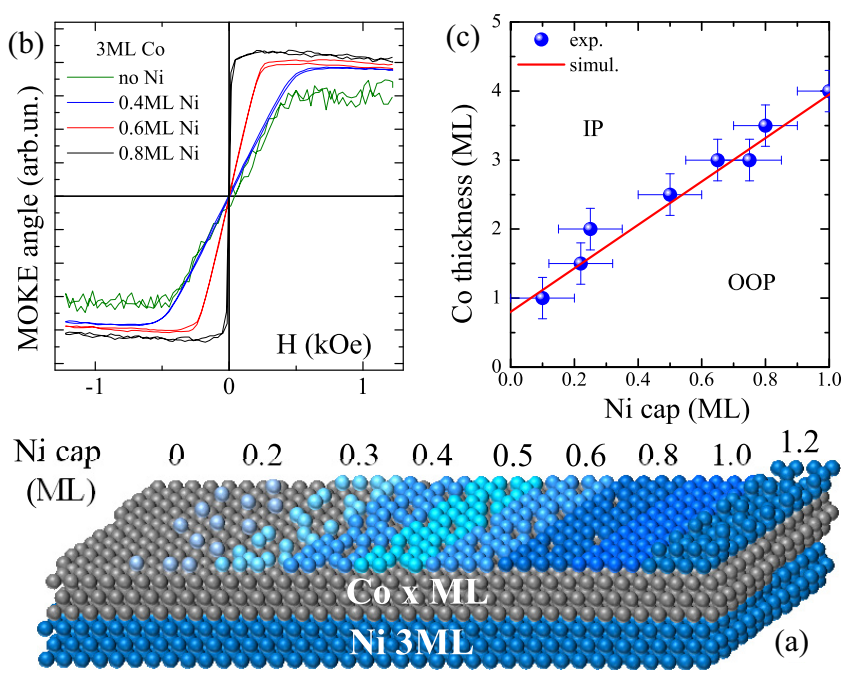

FIG. 3. Co magnetic-moment orientation depending on Ni capping measured by using Kerr magnetometry. (a) Sample stack, (b) hysteresis loops for 3-ML Co with different Ni capping, (c) Ni cap thickness leading to in-plane (IP) to (OOP) out-of-plane magnetization transition for different Co thicknesses. The red curve is a fit using Eq. (2).

easy axis turns from in plane to out of plane for a Co layer thickness $x$ as plotted in Fig. 3(c). The $(x, y)$ values may be determined by writing the total magnetic energy defining the effective anisotropy as [5]

$$
K_{\text {eff }} D=\sum_{\text {atoms } i}\left(K_{S}+K_{V}^{i} t_{i}\right)+K_{\text {shape }} D,
$$

where $K_{S}$ are the interface magnetic anisotropy terms, $K_{V}^{i}$ are the volumetric magnetocrystalline anisotropy of the $i$ th layer of thickness $t_{i}, D$ is the total thickness, and $K_{\text {shape }}$ are the shape anisotropy terms. The transition from in-plane to out-of-plane easy axis corresponds to $K_{\text {eff }}=0$ leading to a unique $(x, y)$ solution. The $(x, y)$ determination is however difficult first because the $K_{\text {shape }}$ term does not vary linearly with $x$ and $y$ and second because several interfaces are involved, i.e., $\mathrm{Co} / \mathrm{Ni}, \mathrm{Co} / \mathrm{MgO}, \mathrm{Ni} / \mathrm{MgO}$, and $\mathrm{Ni} / \mathrm{Au}$ (since the first $\mathrm{Ni}$ layer is grown on $\mathrm{Au}$ ). However a rough estimation can be achieved by considering that $K_{s}^{\mathrm{Co} / \mathrm{MgO}} \approx K_{s}^{\mathrm{Ni} / \mathrm{MgO}}$ (noted $K_{s}^{\mathrm{MgO}}$ in the following). Moreover, the Ni bulk anisotropy term $K_{V}^{\mathrm{Ni}}$ is very small as shown by several groups [15-17] and can be neglected. Considering our stack one can write

$$
\begin{aligned}
K_{\mathrm{eff}} D= & K_{s}^{\mathrm{Co} / \mathrm{Ni}}(1+y)+K_{s}^{\prime}+K_{V}^{\mathrm{Co}} d(x-1) \\
& +K_{\text {shape }} D \\
\text { with } \quad K_{\text {shape }} D= & -\frac{\mu_{o}}{2} \frac{\left(t_{\mathrm{Co}} M_{\mathrm{Co}}+t_{\mathrm{Ni}} M_{\mathrm{Ni}}\right)^{2}}{t_{\mathrm{Co}}+t_{\mathrm{Ni}}}, \\
t_{\mathrm{Co}}= & d x t_{\mathrm{Ni}}=d(3+y) \text { and } \\
K_{s}^{\prime}= & K_{s}^{\mathrm{MgO}}+K_{s}^{\mathrm{Ni} / \mathrm{Au}} .
\end{aligned}
$$

Here $d \cong 0.2 \mathrm{~nm}$ is the distance between atomic planes very similar in $\mathrm{Ni}$ and $\mathrm{Co}$ [15]. A second-order equation on $y$ is obtained parametrized by $x$. Note that the bulk anisotropy term $K_{V}^{\mathrm{Co}}$ is multiplied by $(x-1)$ since there is no bulk contribution for $1 \mathrm{Co}$ atomic plane. In a previous study on a series of $\mathrm{Co} / \mathrm{Ni}(111)$ superlattices, we measured $K_{V}^{\mathrm{Co}}=$ $+0.7 \pm 0.1 \mathrm{MJ} / \mathrm{m}^{3}$ and $K_{s}^{\mathrm{Co} / \mathrm{Ni}}=+0.43 \pm 0.02 \mathrm{~mJ} / \mathrm{m}^{2}[15]$. One should note here that both positive values help PMA. This is the case for $\mathrm{Co} / \mathrm{Ni}$ interface anisotropy and also "bulk" Co magnetocrystalline anisotropy since (111) is an easy axis. A very good fit is obtained [Fig. 3(c)] using $K_{s}^{\prime}=-0.1 \pm$ $0.05 \mathrm{~mJ} / \mathrm{m}^{2}$. From Ref. [38] $K_{s}^{\mathrm{Ni} / \mathrm{Au}}=-0.15 \mathrm{~mJ} / \mathrm{m}^{2}$ was obtained in MBE-grown samples. This means that the $\mathrm{MgO} / \mathrm{Ni}$ and $\mathrm{MgO} / \mathrm{Co}$ interface anisotropies are small and not relevant here.

This analysis allowed us to estimate the number of bilayers necessary to get PMA under vacuum for photoemission experiments on Co terminated layers. For that $K_{s}^{\mathrm{Co} / \mathrm{MgO}}$ and $K_{S}^{\mathrm{Ni} / \mathrm{MgO}}$ should be changed in Eqs. (1) and (2) by $K_{\mathrm{s}}^{\mathrm{Co} / \mathrm{UHV}}$ and $K_{\mathrm{s}}^{\mathrm{Ni} / \mathrm{UHV}}$ given in Ref. [5]. This analysis thus shows that two bilayers $\left(\mathrm{Co}_{x} \mathrm{Ni}_{3 \mathrm{ML}}\right)$ without $\mathrm{Ni}$ cap on top are enough to get PMA up to $x=2-$ ML Co. However, for $x=3 \mathrm{MLs}$ three repeats are necessary to get PMA which was confirmed performing SR-PES experiments.

\section{B. XMCD investigation}

Using the XMCD technique we wanted to address questions raised by a previous XMCD work [15] including (i) what is the influence of the spin magnetic dipole operator on the spin moment determination using the sum rules, and (ii) is the measurement of the orbital moment anisotropy $\Delta m_{\mathrm{L}}$ comparable to the theoretical explanation of PMA proposed by Bruno [36]? In order to answer to these questions the absorption spectra were measured at different angles between the sample and the photon beam, maintaining the saturation magnetization along the beam direction [39]. These conditions require high magnetic fields to be fulfilled. The experiments were performed on the DEIMOS beamline at the SOLEIL synchrotron [40,41]. The setup is equipped with a superconducting coil allowing variable temperature $(1.5-350 \mathrm{~K})$ and high magnetic field measurements (up to $7 \mathrm{~T}$; we applied $4 \mathrm{~T}$ here). Absorption spectra were recorded on a series of $\mathrm{Al}_{2} \mathrm{O}_{3}$ substrate/V/Au/Ni (3 ML)/Co $(x \mathrm{ML}) / \mathrm{Ni}(2 \mathrm{ML}) / \mathrm{Au}$ samples $(x=1,2,3)$ for five angles (noted $\gamma$; see inset in Fig. 4) between the photon/magnetic field direction and the (111) surface normal $\left(\gamma=0,15,30,45\right.$, and $\left.60^{\circ}\right)$.

The top Au layer is a capping layer to avoid oxidation of the top Ni layers. The measured XMCD spectra obtained at the $\mathrm{Co}$ and $\mathrm{Ni}$ edges for the 1-ML Co sample are shown in Fig. 4. The orbital and effective spin moments were deduced using the sum rules and plotted versus $\sin ^{2} \gamma$ [42]. All the absorption spectra were corrected from the saturation effect $[43,44]$. Such experiments allowed us to obtain the orbital moment and moment anisotropy $\Delta m_{L}^{i}(i=\mathrm{Co}, \mathrm{Ni})$ and the spin magnetic dipole operator $T_{z}$ contribution in the spin sum rule as [45]

$$
\begin{gathered}
m_{L}=m_{L}^{\perp}+\left(m_{L}^{\|}-m_{L}^{\perp}\right) \sin ^{2} \gamma=m_{L}^{\perp}-\Delta m_{L} \sin ^{2} \gamma, \\
m_{s}^{\mathrm{eff}}=m_{s}+7 T_{z}=m_{s}+14 Q_{x x}-21 Q_{x x} \sin ^{2} \gamma .
\end{gathered}
$$




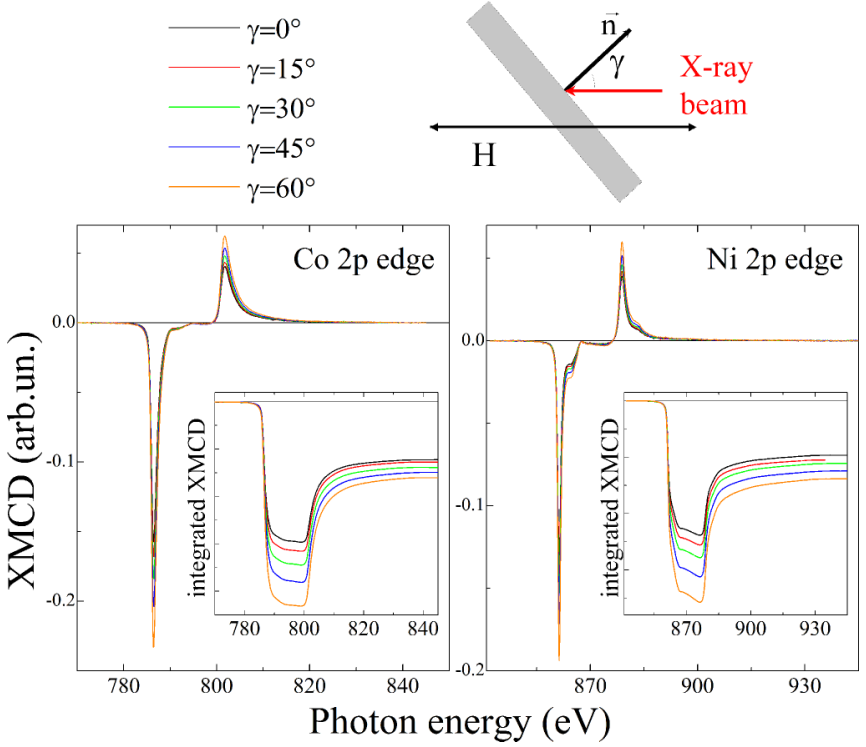

FIG. 4. Examples of XMCD raw data observed at the Co and Ni $L$ edges varying the angle $\gamma$ between the $\mathrm{X}$-ray beam and the surface normal. The magnetic field to get the XMCD is applied along the $\mathrm{x}$-ray beam.

Note that the second equation is derived for a $3 d$ metal with a uniaxial symmetry [46] and shows that the anisotropy of $m_{s}^{\text {eff }}$ is only due to the anisotropy of $T_{z}$. The anisotropy of the orbital and spin effective moment are thus deduced from the slope of the curves $m_{L}\left(\sin ^{2} \gamma\right)$ and $m_{s}^{\text {eff }}\left(\sin ^{2} \gamma\right)$. These measurements were reproduced at 20, 50, 100, 200, and $300 \mathrm{~K}$. The results obtained on the 1-ML Co sample are summarized

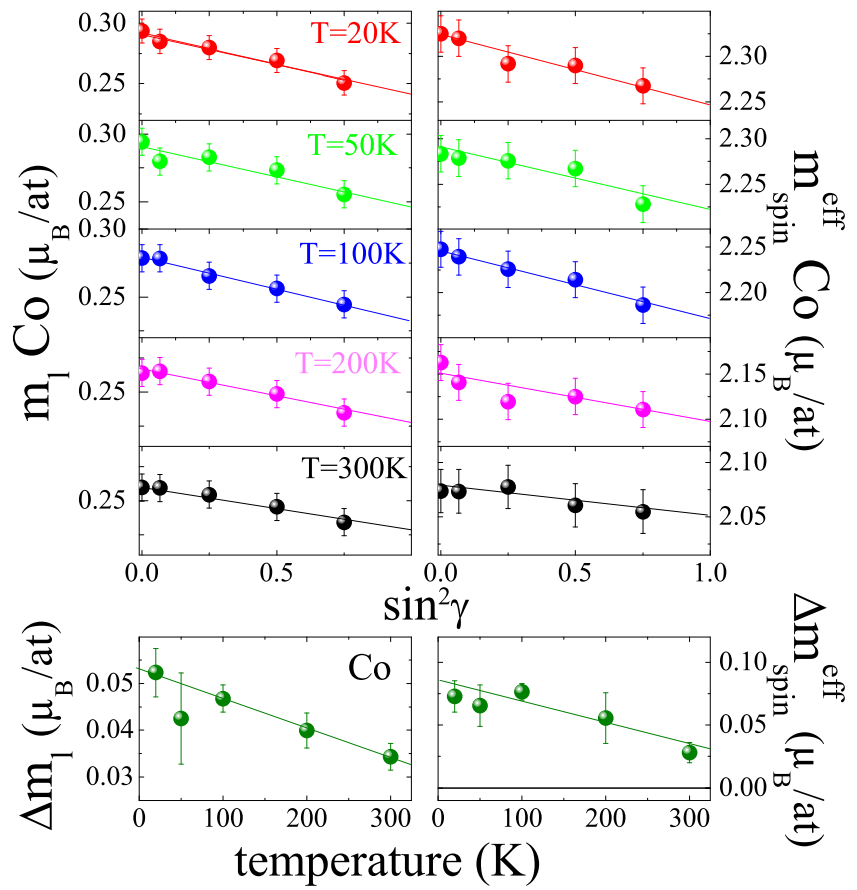

FIG. 5. Top: orbital and spin effective Co magnetic moments measured on the 1-ML Co sample for different $\gamma$ angles and temperatures. Bottom: $m_{L}$ and $m_{s}^{\text {eff }}$ slopes plotted vs temperature.

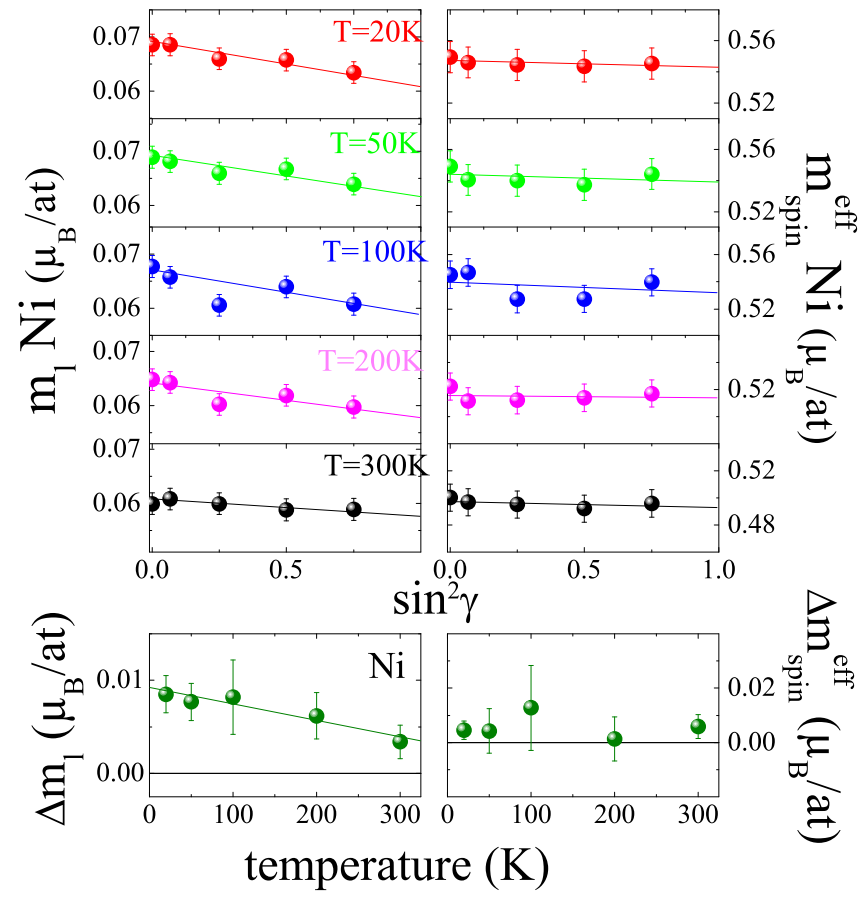

FIG. 6. Similar experiments as in Fig. 5 but for Ni.

in Figs. 5 and 6 for $\mathrm{Co}$ and $\mathrm{Ni}$ absorption edges respectively. It should be noted that $\mathrm{Ni}$ results show larger distribution than Co simply because the Co magnetic moments are around four times larger than $\mathrm{Ni}$ ones. From these results on the 1-ML Co sample, we are first able to estimate the impact of the $T_{z}$ spin magnetic dipole operator on the $\mathrm{Co}$ and $\mathrm{Ni}$ spin moment determination using XMCD by looking at the slope of the $m_{s}^{\text {eff }}\left(\sin ^{2} \gamma\right)$ curves [see Eqs. (3) and (4)]. This is observed negligible for $\mathrm{Ni}$ at all temperatures of measurement whereas

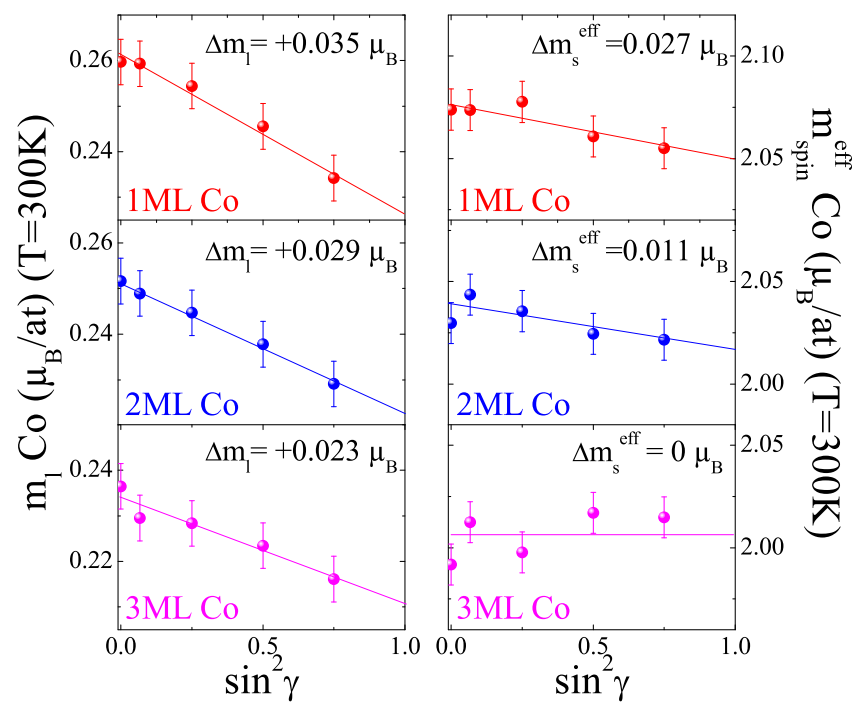

FIG. 7. Co orbital and spin effective moment anisotropies measured at room temperature for three samples with 1-, 2-, and 3-ML Co. The Co PMA and the $T_{z}$ contribution are observed to increase when decreasing the Co thickness. This is consistent with the interfacial origins of both anisotropies. 
a small but measurable $T_{z}$ effect is observed for Co. These two results are consistent with our previous $a b$ initio calculations [15]. This experimental $T_{z}$ amplitude effect is also observed to increase when decreasing the temperature. It remains low even at $0 \mathrm{~K}$ (less than $0.1 \mu_{\mathrm{B}} /$ atom) but large enough to take it into account for a correct $m_{s}$ determination. Second, the PMA is confirmed by the observation of a negative $m_{L}^{\|}-m_{L}^{\perp}$ slope with $\sin ^{2} \gamma$ for both $\mathrm{Co}$ and $\mathrm{Ni}$ atoms. We also observed that this orbital moment anisotropy is increasing when decreasing the temperature as expected. Similar experiments were performed for the 2- and 3-ML Co samples at room temperature. This XMCD analysis at the Co edge is shown in Fig. 7. The $T_{z}$ contribution is observed to almost vanish for 3-ML Co which is not surprising since the $T_{z}$ contribution originates from the interfaces. We also observed that the orbital moment anisotropy decreases when increasing the Co thickness. Again this is consistent with the $\mathrm{Co} / \mathrm{Ni}$ interface origin of the PMA.

These measurements also allow us to determine the gyromagnetic $g$ factor in both $\mathrm{Ni}$ and Co layers. This gives important information on the quality of the XMCD measurements and on the physics of PMA in this system [19,47]). Figure 8 displays the magnetization temperature dependence for the 1and 3-ML-thick Co samples. These magnetization variations are consistent with the reduced Curie temperature $\left(T_{C}\right)$ of such stacks due first to size effects (small thicknesses) and second to different $T_{C}$ in bulk Co and Ni (1388 and $627 \mathrm{~K}$ ). Consequently the smaller is the Co thickness, the smaller the $T_{C}$ of the stack (for the same Ni quantity) and the higher the magnetization variation with temperature.

Finally, the $g$ factors can be extracted by using the equation

$$
\frac{\mu_{l}}{\mu_{s}}=\frac{g-2}{2} .
$$

Although the $g$ factor is an average value over the material as measured for instance by ferromagnetic resonance (FMR) [19], one can extract atomiclike $g$ values from $\mathrm{Ni}$ and Co orbital and spin moments as plotted in Fig. 8. One should notice that

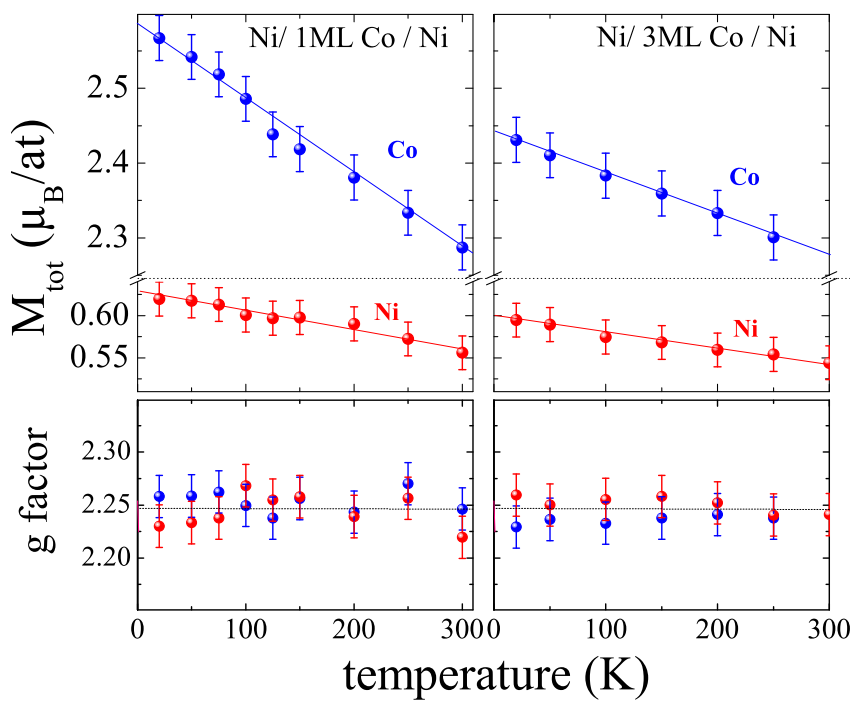

FIG. 8. Top: total $\mathrm{Co}$ and $\mathrm{Ni}$ magnetic moment variation with temperature for the 1-ML Co (left) and 3-ML Co (right) samples. Bottom: the $g$ factors extracted from these data. these extracted $g$ factors are not dependent on temperature as expected. This reinforces the robustness of the measurement and analysis. Even if the accuracy on the $g$ factor is limited due to the addition of errors on $\mathrm{Co}$ and $\mathrm{Ni}$ moments, we find similar values for $\mathrm{Ni}$ and $\mathrm{Co}$, meaning that these atomiclike $g$ factors are similar to those measured by FMR. These $g$ factors are quite far from the 2 value corresponding to free electrons. This is due to the strong $\mathrm{Co} / \mathrm{Ni}$ hybridization at the interface also responsible for the PMA. We find similar $g$-factor values in $\mathrm{Ni}$ and $\mathrm{Co}$ around 2.25 for 1-ML Co at $300 \mathrm{~K}$. This is larger than the value measured for sputtered $\mathrm{Co} / \mathrm{Ni}$ films, around 2.18 $[19,47]$ yet this discrepancy can be readily explained.

Indeed, we have shown that the PMA is slightly lower in sputter grown than in MBE grown films [15] as was confirmed by other groups $[19,25,47]$. The present difference in $g$ factors is another signature of this PMA difference. Finally, it is interesting to compare the orbital moment anisotropies measured separately in $\mathrm{Co}$ and $\mathrm{Ni}$. The anisotropy is six times larger in Co than in $\mathrm{Ni}\left(0.054 \mu_{\mathrm{B}}\right.$ and $0.009 \mu_{\mathrm{B}}$ respectively at $4 \mathrm{~K}$ see Figs. 5 and 6) and one may consider that Ni plays a minor role in this process. Nevertheless, the ratio $m_{L} / m_{s}$ (proportional to the $g$ factor) for $\mathrm{Co}$ and $\mathrm{Ni}$ are similar in $\mathrm{Co}$ and Ni (Fig. 8).

We can go further by looking at the macroscopic magnetic anisotropy link with the microscopic one in order to compare it to Bruno's model. Our XMCD analysis allows us to extract the average $\Delta \mu_{\text {orb }}$ as

$$
\Delta \mu_{l}=\frac{t_{\mathrm{Co}} \Delta \mu_{l}^{\mathrm{Co}}+t_{\mathrm{Ni}} \Delta \mu_{l}^{\mathrm{Ni}}}{t_{\mathrm{Co}}+t_{\mathrm{Ni}}} .
$$

Moreover, we extracted the macroscopic anisotropy $K_{u}$ by measuring the effective anisotropy $K_{\text {eff }}$ on hysteresis loops performed by XMCD applying OOP and IP magnetic field (Fig. 9) and using

$$
K_{u}=K_{\mathrm{eff}}+\frac{1}{2} \mu_{\mathrm{o}} M_{\mathrm{s}}^{2}
$$
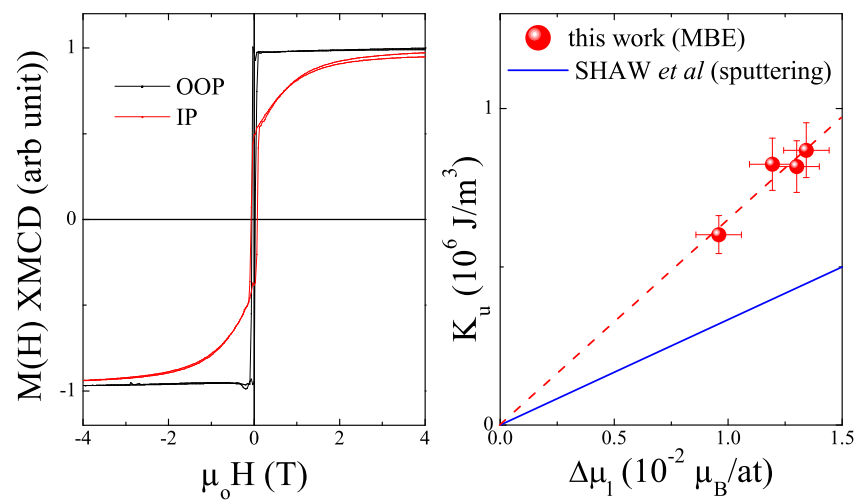

FIG. 9. Left: hysteresis loops measured by XMCD at the Co edge on a 1-ML Co sample for out-of-plane and in-plane applied fields. The area between OOP and IP loops gives the effective anisotropy.Right: resulting macroscopic magnetic anisotropy vs the atomic-orbital moment anisotropy. A linear law is found in agreement with Bruno's model (dashed red line). The blue line comes from results on sputtered samples reported in Ref. [19]. 
According to Bruno's model, these macroscopic and microscopic anisotropies are linked as $[19,35]$

$$
K_{u}=A \frac{\xi N}{4 V} \frac{\Delta \mu_{l}}{\mu_{B}},
$$

where $N / V$ is the atomic density (close to $8.9 \times$ $10^{28}$ atom $/ \mathrm{m}^{3}$ here), $\xi$ the spin-orbit coupling parameter (we use the same value as in [19], $\xi \approx-1.6 \times 10^{-20} \mathrm{~J} /$ atom), $\mu_{B}$ the Bohr magneton, and $A$ is a prefactor found in the literature to vary between 0.05 and 0.2 [41,47]. We report in Fig. 9 our data together with the results obtained on sputtered multilayer samples by Shaw's group [19]. We also confirm Bruno's law but interestingly, we obtained a slope $A=0.18$ around two times larger than those obtained on sputtered samples.

\section{CRYSTALLINE ARRANGEMENT STUDIED BY SURFACE DIFFRACTION}

The previously published disagreements obtained between $a b$ initio magnetic-moment calculations and the experimental results $[15,25,33]$ motivated us to re-examine the crystallographic ordering in $\mathrm{Co} / \mathrm{Ni}$ superlattices. Previous calculations were carried out by considering a perfect fcc stack of hexagonal lattices, with distances between atoms similar or very close to those of the bulk phases. On the one hand, the distance between the $\mathrm{Co}$ and $\mathrm{Ni}$ planes at the interface may impact the electronic properties if, for example, it is much different compared to bulk interplanar distance used in the calculations. Moreover, a particular arrangement of the $\mathrm{Co}$ and $\mathrm{Ni}$ atoms at the interface has to be considered. To get such information, we performed surface x-ray-diffraction experiments on Co films. We first examined Co films deposited on the $\mathrm{Al}_{2} \mathrm{O}_{3} / \mathrm{V} / \mathrm{Au} / \mathrm{Ni}(111)$ stack but in that case we increase the number of parameters in the fit of the truncation rods. We thus eliminated this complexity by analyzing the Co structure when deposited on a single-crystalline $\mathrm{Ni}(111)$ substrate. We also examined Co films covered with a Ni ML.

In a surface diffraction experiment, the $\mathrm{x}$-ray beam is sent at grazing incidence. The diffraction pattern consists in surface truncation rods perpendicular to the surface in the reciprocal space. A surface diffraction experiment consists of measuring these rods. Their profiles are extremely sensitive to various factors: atomic positions in the lattice, the type of atoms at each site, the stacking sequence, distances in the plane and out of the plane, and roughness [48-50]. These experiments generally require the use of synchrotron radiation given the strong reduction of the signal compared to conventional diffraction setup. Moreover, the energy choice using synchrotron radiation is mandatory in the present case since $\mathrm{Ni}$ and $\mathrm{Co}$ are neighbors in the periodic table so that their x-ray diffusion contrast is then very low using a regular $\mathrm{x}$-ray-diffraction setup. One elegant way of overcoming this difficulty is to set the x-ray beam energy at the $1 s$ Co edge $(h v=7709 \mathrm{eV})$. The Co scattering factor is then strongly decreased by about 12 electrons whereas that of $\mathrm{Ni}$ remains close to 26 electrons at this energy, thus ensuring a high diffraction contrast to $\mathrm{x}$ rays (a procedure called anomalous surface diffraction).

The experiments were carried out on the UHV diffraction station of the SixS beamline at the SOLEIL synchrotron source. The samples were prepared in the preparation chamber hosted
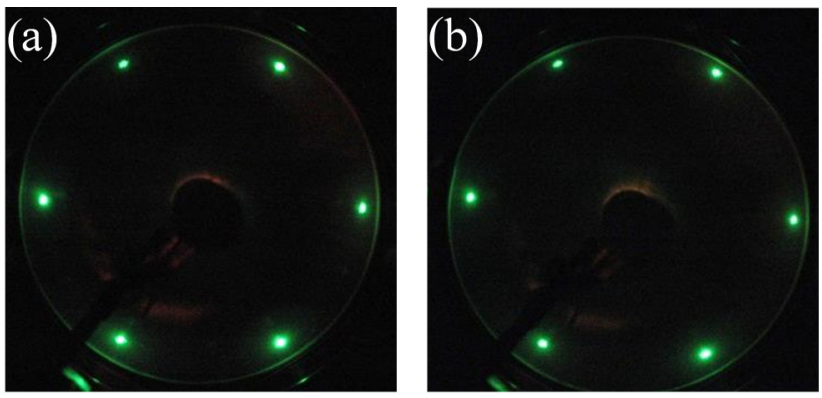

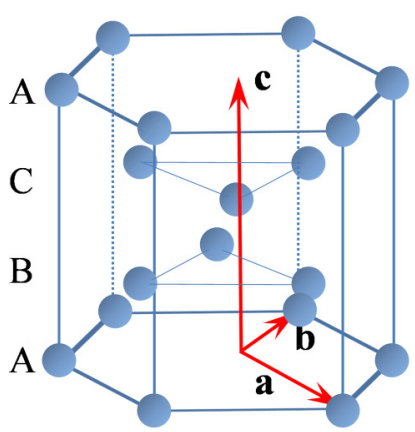

(c) unit cell truncation rods

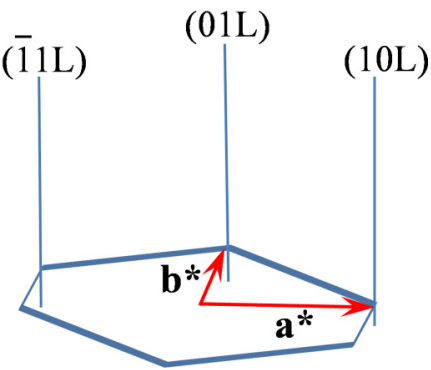

(d) reciprocal lattice
FIG. 10. LEED patterns obtained on (a) the Ni(111) surface and (b) after the growth of 2-ML-thick Co film. The unit cell chosen for ROD simulation is indicated in (c). Some truncation rods indexation is shown in (d). Six rods were measured for each sample.

by the diffractometer. The cleaning process of the substrate surface involved various cycles of $\mathrm{Ar}+$ ion sputtering at $1 \mathrm{keV}$ and subsequent annealing to $1100 \mathrm{~K}$. The cleanliness and surface quality of the samples was checked by means of Auger electron spectroscopy and low-energy electron diffraction (LEED), respectively. Co was deposited using an $e$-beam evaporator. The Co deposition rate was calibrated using a quartz microbalance located at the place of the sample. Co was deposited at room temperature (to avoid intermixing) at a rate of $1 \mathrm{ML} / \mathrm{min}$. For each sample the $\mathrm{Ni}(111)$ substrate was reprepared following the above-mentioned procedure. Figures 10(a) and 10(b) show the LEED pattern measured for the clean Ni(111) surface and after the Co deposition, respectively. Six different samples were prepared and analyzed: the bare $\mathrm{Ni}(111)$ substrate, $x$ ML $\mathrm{Co} / \mathrm{Ni}(111)$ substrate with $x=1,2$, and 3 , and $1 \mathrm{ML} \mathrm{Ni} / x \mathrm{ML}$ $\mathrm{Co} / \mathrm{Ni}(111)$ with $x=1$ and 2 .

The $\mathrm{x}$-ray-diffraction data presented in this paper are indexed according to the surface unit cell of $\mathrm{Ni}(111)$ described by the following parameters: $a=b=0.2489 \mathrm{~nm}, c=0.609 \mathrm{~nm}$ and $\alpha=\beta=90^{\circ}, \gamma=120^{\circ}$. Indeed, since Co can be grown in fcc (ABCABC stacking sequence) or in hcp (ABAB stacking), at least four planes stacked along (111) were considered to account for these two possible types of stacking [Fig. 10(c)]. A schematic view of the reciprocal space is represented in Fig. 10(d). For each sample we measured the integrated intensities along six crystal truncation rods on each sample: $(11 L)$, $(1-2 L),(-10 L),(1-1 L),(-11 L),(0-1 L)$ with $L$ varying from 0.1 to 2.4 (larger values of $L$ were not achievable due to geometric constraints). This gives three series of nonequivalent 


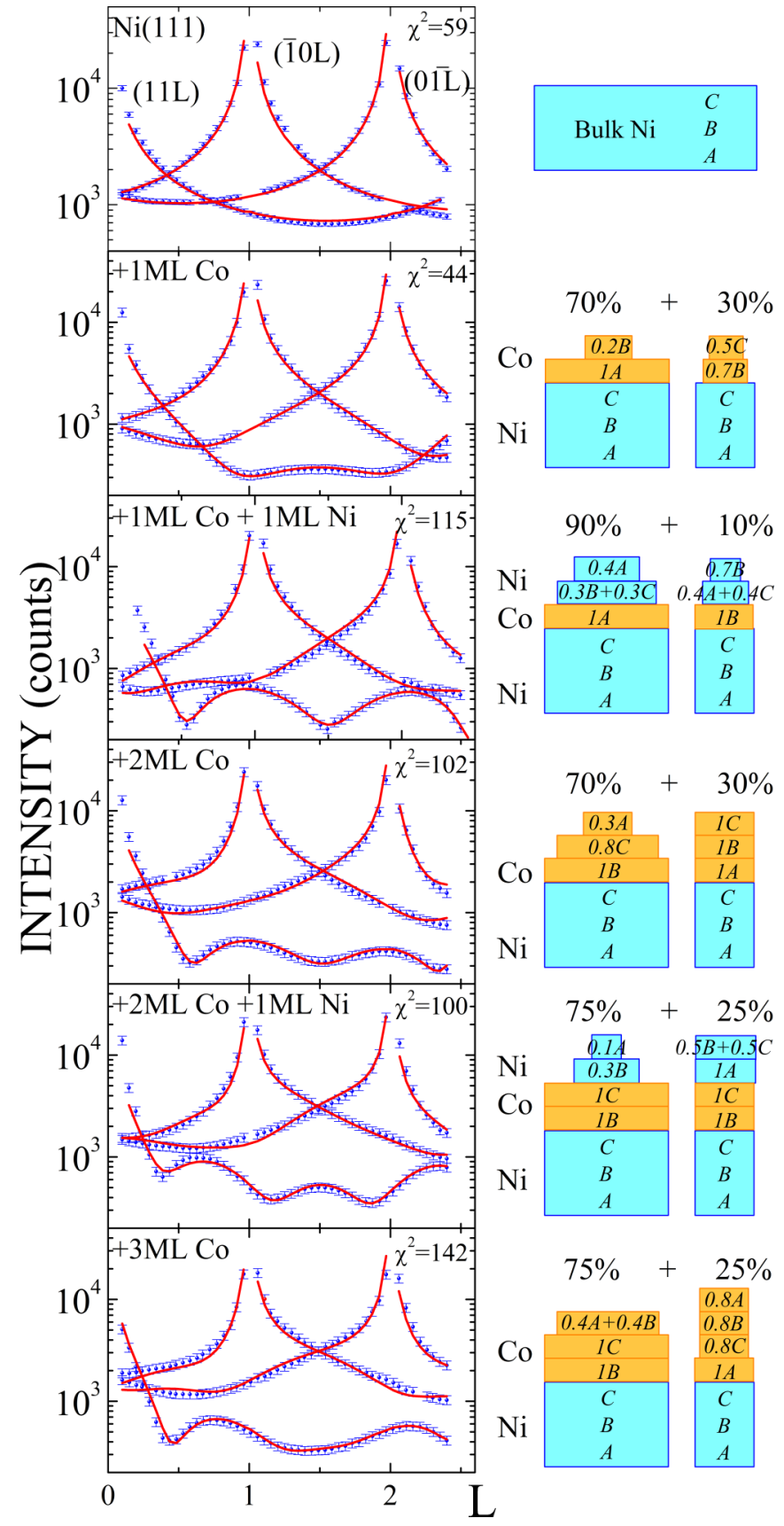

FIG. 11. Experimental structure factors (blue open circles, together with error bars) and the best-fit structure factors (solid red line) truncations rods and simulation for the six samples, from top to bottom: Ni(111) substrate, $\mathrm{Ni}+1-\mathrm{ML} \mathrm{Co}, \mathrm{Ni}+1-\mathrm{ML} \mathrm{Co}+1-\mathrm{ML}$ $\mathrm{Ni}, \mathrm{Ni}+2-\mathrm{ML} \mathrm{Co}, \mathrm{Ni}+2-\mathrm{ML} \mathrm{Co}+1-\mathrm{ML} \mathrm{Ni}, \mathrm{Ni}+3-\mathrm{ML}$ Co. The schematic representation of the models of stacks obtained after ROD simulations is shown on the right.

truncation rods along (Fig. 10)

(i) (11L), $(1-2 \mathrm{~L})-$ Bragg peak at $\mathrm{L}=0$,

(ii) $(-10 \mathrm{~L}),(1-1 \mathrm{~L})-$ Bragg peak at $\mathrm{L}=1$,

(iii) $(-11 \mathrm{~L}),(0-1 \mathrm{~L})-$ Bragg peak at $\mathrm{L}=2$.

After background subtraction and application of standard correction factors a fit of the data was carried out using the software package ROD [51].
A detailed structural refinement was performed by fitting simultaneously three nonequivalent truncation rods. The experimental (blue circles) together with the best fit (red curve) structure factors are shown in Fig. 11. During the refinement, the existence of two domains with different stacks had to be considered, whose percentages are also given on the right-hand side of Fig. 11. It should be noted that in all the simulations the extracted roughness was found very close to zero (the simulations are shown for a roughness equal to zero). For the Ni substrate, during the refinement the only variable is the out-of-plane relaxation of the topmost plane. The simulations are excellent and almost no surface relaxation is observed $(<1 \%)$ in agreement with the literature [52].

For the 1-ML Co film, the nucleation site of the Co layer must be taken into account during the refinement. Considering the $\mathrm{ABC}$ stacking of the Ni substrate by terminating with a $\mathrm{C}$ plane, several options may be considered for Co nucleation: the nucleation site (A, B, or C) is left free for both domains. The results of the refinement show that the site $C$ is not occupied and that sites A (domain 1) and B (domain 2) can be occupied with some preference for site A. The fit also shows that the Co film thickness is not exactly $1 \mathrm{ML}$ but $1.2 \mathrm{MLs}$, which is within the error bar of the thickness control on this setup. Therefore a second plane has grown on the first monolayer. Two types of stacking are obtained corresponding to $70 \%$ as an extension of the fcc structure of $\mathrm{Ni}$ and to $30 \%$ as a hcp stacking. When this type of deposit is covered with $1 \mathrm{ML}$ of $\mathrm{Ni}$, the fcc stacking is then favored representing $90 \%$ of the surface.

The situation changes drastically for Co thickness higher than 1 ML. For 2 MLs thick, the Co film adopts preferentially the hcp structure (domain 1, 70\%), even if there remain zones with an fcc stacking (domain 2, 30\%). For 3-ML Co, the hcp stacking dominates and no more fcc stacking occurs. The Ni capping no longer affects the stacking of the Co which remains hcp, contrary to the previous case with a single plane of Co. Finally, the interplanar distances for all these samples obtained following the data refinements are found very close to the bulk $\mathrm{Ni}$ atomic distance. The out-of-plane relaxations never exceed $+/-3 \%$, which is of the order of magnitude of the misfit between $\mathrm{Co}$ and $\mathrm{Ni}$ bulk lattices.

Therefore this analysis clearly demonstrates that there is no special atomic arrangement other than the fcc or hcp stacking, and no strong lattice parameter relaxation. The only parameter that changes from one sample to another is the stacking sequence. For 1-ML Co film the fcc stacking is preferred, but the hcp stacking dominates for Co films thicker than 2 MLs. This means that an atomic plane of Co needs to be covered by Co to generate the hcp structure.

\section{AB INITIO CALCULATIONS}

In the previous section, we finally observed that many Co stacks on Ni between fcc and hcp may occur. Up to now the $a b$ initio calculations were performed only considering a fcc stack in the whole superlattice [22]. The electronic structure of the $\mathrm{Ni}(3 \mathrm{ML}) / \mathrm{Co}(2 \mathrm{ML})$ superlattices with different stacking of the $\mathrm{Ni}$ and $\mathrm{Co}$ monolayers have thus been calculated from first principles, using the code WIEN2K [53] and the Perdew-BurkeErnzerhof (PBE) [54] functional for describing the exchange and correlation potential. We used atomic spheres with a radius 
TABLE I. Calculated spin magnetic moment of $\mathrm{Ni}$ and $\mathrm{Co}$ atoms at the interfaces and of $\mathrm{Ni}$ atoms at the center of the $\mathrm{Ni}$ layers in $\mathrm{Ni}(3$ $\mathrm{ML}) / \mathrm{Co}(2 \mathrm{ML})$ superlattices with different stacking sequences. The averaged value of the nickel spin atomic moment is also given. In the last line are reported the experimental spin moments of a 1-ML Co in between Ni at $10 \mathrm{~K}$ given by XMCD.

\begin{tabular}{|c|c|c|c|c|}
\hline \multirow[b]{2}{*}{ Superlattices stacking } & \multicolumn{4}{|c|}{ Theoretical spin magnetic moment (Bohr magneton) } \\
\hline & $\begin{array}{c}\mathrm{Ni} \\
\text { interface }\end{array}$ & $\begin{array}{c}\mathrm{Ni} \\
\text { central }\end{array}$ & $\begin{array}{c}\mathrm{Ni} \\
\text { average }\end{array}$ & Co \\
\hline $\mathrm{Ni}(\mathrm{fcc}) / \mathrm{Co}(\mathrm{fcc})$ & 0.6784 & 0.6387 & 0.6651 & 1.7426 \\
\hline $\mathrm{Ni}(\mathrm{fcc}) / \mathrm{Co}(\mathrm{hcp}) 1$ & 0.6994 & 0.5997 & 0.6662 & 1.7423 \\
\hline $\mathrm{Ni}(\mathrm{fcc}) / \mathrm{Co}(\mathrm{hcp}) 2$ & 0.6983 & 0.6174 & 0.6713 & 1.7493 \\
\hline \multirow[t]{2}{*}{$\mathrm{Ni}(\mathrm{fcc}) / \mathrm{Co}(\mathrm{hcp})$} & 0.7091 & 0.6289 & 0.6824 & 1.7510 \\
\hline & \multicolumn{2}{|c|}{ Expt. $\mu_{\text {spin }} 10 \mathrm{~K}$} & 0.55 & 2.28 \\
\hline
\end{tabular}

of $0.1164 \mathrm{~nm}$ (2.2 atomic units), an in-plane lattice parameter of $0.2507 \mathrm{~nm}$, and a distance of $0.2047 \mathrm{~nm}$ between successive atomic layers (measured on our samples [15]). We considered four different stackings, which differ by the sequences of the successive $\mathrm{Ni}$ and Co monolayers occupying the conventional $\mathrm{A}, \mathrm{B}$, or $\mathrm{C}$ atomic sites of the fcc lattice. The four superlattices that we studied correspond to the following:

(i) a perfect fcc stacking, labeled " $\mathrm{Ni}(\mathrm{fcc}) / \mathrm{Co}(\mathrm{fcc})$," with the sequence $\mathrm{Ni}(\mathrm{ABC}) / \mathrm{Co}(\mathrm{AB}) / \mathrm{Ni}(\mathrm{CAB}) / \mathrm{Co}(\mathrm{CA}) / \mathrm{Ni}(\mathrm{BCA}) /$ $\mathrm{Co}(\mathrm{BC})$,

(ii) a first stacking combining fcc $\mathrm{Ni}$ and hcp $\mathrm{Co}$, labeled "Ni(fcc)/Co(hcp)1," with the sequence $\mathrm{Ni}(\mathrm{ABC}) / \mathrm{Co}(\mathrm{BC}) /$ $\mathrm{Ni}(\mathrm{BCA}) / \mathrm{Co}(\mathrm{CA}) / \mathrm{Ni}(\mathrm{CAB}) / \mathrm{Co}(\mathrm{AB})$,

(iii) a second stacking combining fcc $\mathrm{Ni}$ and hep Co, labeled "Ni(fcc)/Co(hcp)2," with the sequence $\mathrm{Ni}(\mathrm{ABC}) /$ $\mathrm{Co}(\mathrm{AC})$

(iv) finally a perfect hcp stacking, labeled "Ni(hcp)/ $\mathrm{Co}(\mathrm{hcp})$," with the sequence $\mathrm{Ni}(\mathrm{ABA}) / \mathrm{Co}(\mathrm{BA}) / \mathrm{Ni}(\mathrm{BAB}) /$ $\mathrm{Co}(\mathrm{AB})$.

The spin magnetic moments that we calculated for the different atoms in these SLs are given in Table I. To summarize, the stacking sequence has a small influence on the averaged nickel magnetic moment and on the Co spin magnetic moment, which only vary by $2.6 \%$ and $0.5 \%$, respectively. Finally, we also varied the distance at the interface between $\mathrm{Co}$ and $\mathrm{Ni}$ up to $5 \%$ and the Co atomic moment was observed to vary insignificantly compared to our observations.

\section{DISCUSSION}

The first point we want to address concerns the link between macroscopic anisotropy via $K_{u}$ and microscopic anisotropy via $\Delta m_{l}$ (Sec. III B, Fig. 9). The agreement with Bruno's model is quite comparable to that observed in other epitaxial systems. However, this law is not similar for epitaxial $\mathrm{Co} / \mathrm{Ni}$ superlattices (MBE) or for multilayers (sputtering). This observation is not surprising since at least four different groups $[15,19,25,47]$ came to the same conclusion: the macroscopic magnetic anisotropy $K_{u}$ is higher in epitaxial films. However, the anisotropy on the atomic-orbital moment $\left(\Delta m_{l}\right)$ observed here is similar to the values reported by Shaw and co-workers [19]. This strongly suggests that the origin of the $K_{u}$ difference obtained between epitaxial and sputtered samples does not occur at the atomic level but on a larger scale (possibly roughness or small impurities concentration).
Concerning the atomic magnetic moments (Sec. III B), the spin moment of $\mathrm{Ni}$ is therefore close to the bulk value, whereas a noticeable increase of the orbital moment is observed here compared to the bulk. The situation is more complex for Co. The previous XMCD results [15] indicate that two different sites (and only two) need to be considered in the stack of Co layers. At the interface with $\mathrm{Ni}$, the two contributions of spin and orbit are strongly increased with respect to bulk (hcp or fcc) Co values. On the other hand, for the Co atoms surrounded by Co (the central plane for a layer of three planes for example), the measured moments are then comparable to the bulk values. The strong increase of the Co moments at the interface with $\mathrm{Ni}$ had previously been discussed [15] as possibly coming from the application of the sum rules in particular for the spin moment determination. Indeed, the contribution of $T_{z}$ (which can strongly increase at a symmetry-breaking interface) could affect the estimation of the spin moment. In this study, it was possible to determine experimentally this contribution. We find it to be low even if it is not negligible. The "true" values of the spin moments were thus determined. However, they are still increased by about $30 \%$ compared to a whole Co environment, whereas a $6 \%$ increase is given by the calculations considering a perfect fcc structure of superlattices [15]. We have therefore carried out calculations by considering an hcp stack of Co (Sec. V, Table I). The results remain unchanged. Finally, we have varied in the calculations the distances between the plane of $\mathrm{Co}$ at the interface with $\mathrm{Ni}$ and in the Co layers of several \%, in any case beyond what we determined using anomalous surface diffraction. Again, we did not get any increase in Co moments as observed experimentally.

Consequently, there is a significant disagreement between the Co moments determined experimentally and the calculated ones by considering a crystallographic structure in agreement with the observation given by diffraction. This disagreement is surprising since a very simple model gives a qualitative explanation for the observations. Indeed, one can consider on the one hand that at the interface $\mathrm{Co}$ and $\mathrm{Ni}$ exchange electrons in such a way that the $3 d$ band of Co is filled with 7.5 electrons $[15,33]$. On the other hand, if we consider that the $3 d$ band of the majority electrons is completely filled at the interface, there remain 2.5 electrons in the minority band. This scenario then leads to a full spin polarization and to an atomic moment of $2.5 \mu_{\mathrm{B}}$ for $\mathrm{Co}$ at the interface with $\mathrm{Ni}$, in agreement with the experiment. 


\section{CONCLUSION}

In this paper, we show that very high spin polarization is obtained in $\mathrm{Co} / \mathrm{Ni}(111)$ superlattices, a $3 d$ metals based system. We also brought another experimental proof that the PMA in $\mathrm{Co} / \mathrm{Ni}$ superlattices is closely linked to the $\mathrm{Co} / \mathrm{Ni}$ interface. Interestingly, the effective magnetic anisotropy can be tuned by controlling the Ni coverage on a Co film. Such a possibility may be an opportunity for switching the magnetization by an external electric field (in a tunnel barrier for instance), using an adequate $\mathrm{Ni}$ coverage to get the system very close to the inplane to out-of-plane transition. The angle-dependent XMCD analysis using strong field and low temperature allowed us to confirm the very high magnetic moment of Co in contact with $\mathrm{Ni}\left(2.55 \mu_{\mathrm{B}}\right.$ to be compared to the bulk value $\left.1.7 \mu_{\mathrm{B}}\right)$. Such a strong enhancement has still to be reproduced using $a b$ initio calculations but our surface diffraction results drastically limit the number of configurations that have to be considered. The anisotropy of the orbital magnetic moment is shown to be linked to the magnetocrystalline anisotropy following Bruno's model. As a consequence, the much stronger orbital moment anisotropy in Co compared to Ni demonstrates that PMA is essentially due to Co. This analysis also sheds some light on the
PMA difference observed in sputtered and MBE-grown samples. The anisotropy at the atomic level $\left(\Delta \mu_{l}\right)$ are similar using both growth techniques, whereas the macroscopic anisotropy $\left(K_{u}\right)$ is lower on sputtered films. This strongly suggests that the origin of this magnetic anisotropy difference is not at the atomic level but on a large scale. Finally, these results, Co termination to get high $\mathrm{SP}$ and number of $\mathrm{Co} / \mathrm{Ni}$ interfaces to get PMA, have to be taken into account when defining the architecture of a device. This is an example of approaching full spin polarization by exploiting ultrathin transition metal layers and opens interesting directions engineering spintronic materials.

\section{ACKNOWLEDGMENTS}

This work was supported partly by the French PIA project "Lorraine Université d'Excellence," reference ANR15-IDEX-04-LUE. The theoretical calculations were granted access to the HPC resources of CALMIP supercomputing center under the allocation P1252. We acknowledge E. E. Fullerton from the Center for Memory and Recording Research (University of California San Diego) for his critical reading of the manuscript.
[1] J. C. Sankey, Y.-T. Cui, J. Z. Sun, J. C. Slonczewski, R. A. Buhrman, and D. C. Ralph, Measurement of the spin-transfertorque vector in magnetic tunnel junctions, Nat. Phys. 4, 67 (2007).

[2] J. S. Meena, M. S. Simon, C. Umesh, and T-Y Tseng, Overview of emerging nonvolatile memory technologies, Nanoscale Res. Lett. 9, 526 (2014).

[3] T. Silva and W. Rippard, Developments in nano-oscillators based upon spin-transfer point-contact devices, J. Magn. Magn. Mater. 320, 1260 (2010).

[4] K. Dumesnil and S. Andrieu, Molecular Beam Epitaxy: From Quantum Wells to Quantum Dots. From Research to Mass Production Chap 20: Epitaxial Magnetic Layers Grown by MBE: Model Systems to Study the Physics in Nanomagnetism and Spintronic, edited by M. Henini (Elsevier, New York, 2012).

[5] M. T. Johnson, P. J. H Bloemen, F. J. A den Broeder, and J. J. de Vries, Magnetic anisotropy in metallic multilayers, Rep. Prog. Phys. 59, 1409 (1996).

[6] G. Andersson, T. Burkert, P. Warnicke, M. Björck, B. Sanyal, C. Chacon, C. Zlotea, L. Nordström, P. Nordblad, and O. Eriksson, Perpendicular Magnetocrystalline Anisotropy in Tetrago-nally Distorted Fe-Co Alloys, Phys. Rev. Lett. 96, 037205 (2006).

[7] S. Ouazi, S. Vlaic, S. Rusponi, G. Moulas, P. Buluschek, K. Halleux, S. Bornemann, S. Mankovsky, J. Minar, J. B. Staunton, H. Ebert, and H. Brune, Atomic-scale engineering of magnetic anisotropy of nanostructures through interfaces and interlines, Nat. Commun. 3, 1313 (2012).

[8] A. Rajanikanth, S. Kasai, N. Ohshima, and K. Ohno, Spin polarization of currents in $\mathrm{Co} / \mathrm{Pt}$ multilayer and $\mathrm{Co}-\mathrm{Pt}$ alloy thin films, Appl. Phys. Lett. 97, 022505 (2010).

[9] Y. Sakuraba, M. Hattori, M. Oogane, Y. Ando, H. Kato, A. Sakkuma, T. Miyazaki, and H. Kubota, Giant tunneling magnetoresistance in $\mathrm{Co}_{2} \mathrm{Mn} \mathrm{Si} / \mathrm{Al}-\mathrm{O} / \mathrm{Co}_{2} \mathrm{MnSi}$ magnetic tunnel junctions, Appl. Phys. Lett. 88, 192508 (2006).

[10] T. Marukame, T. Ishikawa, K. Matsuda, T. Uemura, and M. Yamamoto, High tunnel magnetoresistance in fully epitaxial magnetic tunnel junctions with a full-Heusler alloy $\mathrm{Co}_{2} \mathrm{Cr}_{0.6} \mathrm{Fe}_{0.4} \mathrm{Al}$ thin film, Appl. Phys. Lett. 88, 262503 (2006).

[11] N. Tezuka, N. Ikeda, S. Sugimoto, and K. Inomata, 175\% tunnel magnetoresistance at room temperature \& high thermal stability using $\mathrm{Co}_{2} \mathrm{FeAl}_{0.5} \mathrm{Si}_{0.5}$ full-Heusler alloy electrodes, Appl. Phys. Lett. 89, 252508 (2006).

[12] S. Andrieu, A. Neggache, T. Hauet, T. Devolder, A. Hallal, M. Chshiev, A. M. Bataille, P. Le Fevre, and F. Bertran, Direct evidence for minority spin gap in the $\mathrm{Co}_{2} \mathrm{MnSi}$ Heusler compound, Phys. Rev. B 93, 094417 (2016).

[13] B. Pradines, R. Arras, I. Abdallah, N. Biziere, and L. Calmels, First-principles calculation of the effects of partial alloy disorder on the static and dynamic magnetic properties of $\mathrm{Co}_{2} \mathrm{MnSi}$, Phys. Rev. B 95, 094425 (2017).

[14] L. You, R. C. Sousa, S. Bandiera, B. Rodmacq, and B. Dieny, $\mathrm{Co} / \mathrm{Ni}$ multilayers with perpendicular anisotropy for spintronic device applications, Appl. Phys. Lett. 100, 172411 (2012).

[15] M. Gottwald, S. Andrieu, F. Gimbert, E. Shipton, L. Calmels, C. Magen, E. Snoeck, M. Liberati, T. Hauet, E. Arenholz, S. Mangin, and E. E. Fullerton, $\mathrm{Co} / \mathrm{Ni}(111)$ superlattices studied by microscopy, X-ray absorption and ab-initio calculations, Phys. Rev. B 86, 014425 (2012).

[16] M. T. Johnson, J. J. de Vries, N. W. E. McGee, J. aan de Stegge, and F. J. A. den Broeder, Orientational Dependence of the Interface Magnetic Anisotropy in Epitaxial Ni/Co/Ni, Phys. Rev. Lett. 69, 3575 (1992).

[17] Y. B. Zhang, J. A. Woollam, Z. S. Shan, J. X. Shen, and D. J. Sellmyer, Anisotropy and magneto-optical properties of 
sputtered Co/Ni multilayer thin films, IEEE Trans. Magn. 30, 4440 (1994).

[18] S. Girod, M. Gottwald, S. Andrieu, S. Mangin, J. McCord, Eric E. Fullerton, J.-M. L. Beaujour, B. J. Krishnatreya, and A. D. Kent, Strong perpendicular magnetic anisotropy in $\mathrm{Ni} / \mathrm{Co}(111)$ single crystal superlattices, Appl. Phys. Lett. 94, 262504 (2009).

[19] J. M. Shaw, H. T. Nembach, and T. J. Silva, Measurement of orbital asymmetry and strain in $\mathrm{Co}_{90} \mathrm{Fe}_{10} / \mathrm{Ni}$ multilayers and alloys: Origins of perpendicular anisotropy, Phys. Rev. B 87, 054416 (2013).

[20] G. H. O. Daalderop, P. J. Kelly, and F. J. A. den Broeder, Prediction and Confirmation of Perpendicular Magnetic Anisotropy in Co/Ni Multilayers, Phys. Rev. Lett. 68, 682 (1992).

[21] K. Kyuno, J. G. Ha, R. Yamamoto, and S. Asano, Perpendicular magnetic anisotropy of metallic multilayers composed of magnetic layers only/ Ni/Co and Ni/Fe multilayers, Jpn. J. Appl. Phys. 35, 2774 (1996).

[22] F. Gimbert and L. Calmels, First-principles investigation of the magnetic anisotropy and magnetic properties of $\mathrm{Co} / \mathrm{Ni}(111)$ superlattices, Phys. Rev. B 86, 184407 (2012).

[23] J.-M. Beaujour, W. Chen, K. Krycka, C.-C. Kao, J. Z. Sun, and A. D. Kent, Ferromagnetic resonance study of sputtered $\mathrm{Co} \mid \mathrm{Ni}$ multilayers, Eur. Phys. J. B 59, 475 (2007).

[24] W. Chen, J.-M. L. Beaujour, G. deLoubens, and A. D. Kent, Spin-torque driven ferromagnetic resonance of $\mathrm{Co} / \mathrm{Ni}$ synthetic layers in spin valves, Appl. Phys. Lett. 92, 012507 (2008).

[25] T. Seki, J. Shimada, S. Iihama, M. Tsujikawa, T. Koganezawa, A. Shioda, T. Tashiro, W. Zhou, S. Mizukami, M. Shirai, and K. Takanashi, Magnetic anisotropy and damping for monolayercontrolled Co|Ni epitaxial multilayer, J. Phys. Soc. Jpn. 86, 074710 (2017).

[26] S. Mangin, D. Ravelosona, J. A. Katine, M. J. Carey, B. D. Terris, and E. E. Fullerton, Current-induced magnetization reversal in nanopillars with perpendicular anisotropy, Nat. Mater. 5, 210 (2006).

[27] S. Mangin, Y. Henry, D. Raveloson, J. A. Katine, and E. E. Fullerton, Reducing the critical current for spin-transfer switching of perpendicularly magnetized nanomagnets, Appl. Phys. Lett. 94, 012502 (2009).

[28] D. P. Bernstein, B. Bräuer, R. Kukreja, J. Stöhr, T. Hauet, J. Cucchiara, S Mangin, J. A. Katine, T. Tyliszczak, K. W. Chou, and Y. Acremann, Nonuniform switching of the perpendicular magnetization in a spin-torque-driven magnetic nanopillar, Phys. Rev. B 83, 180410(R) (2011).

[29] H. Tanigawa, T. Koyama, G. Yamada, D. Chiba, S. Kasai, S. Fukami, T. Suzuki, N. Ohshima, and N. Ishiwata, Domain wall motion induced by electric current in a perpendicularly magnetized Co/Ni nano-wire, Appl. Phys. Express 2, 053002 (2009).

[30] K. Ueda, T. Koyoma, R. Hiramatsu, D. Chiba, S. Fukami, H. Tanigawa, T. Suzuki, N. Ohshima, N. Ishiwata, Y. Nakatani, K. Kobayashi, and T. Ono, Temperature dependence of carrier spin polarization determined from current-induced domain wall motion in a Co/Ni nanowire, Appl. Phys. Lett. 100, 202407 (2012).

[31] S. Legal, N. Vernier, F. Montaigne, M. Gottwald, D. Lacour, M. Hehn, J. McCord, D. Ravelosona, S. Mangin, S. Andrieu, and T. Hauet, Thermally activated domain wall motion in [Co/Ni](111) superlattices with perpendicular magnetic anisotropy, Appl. Phys. Lett. 106, 062406 (2015).
[32] S. Le Gall, N. Vernier, F. Montaigne, A. Thiaville, J. Sampaio, D. Ravelosona, S. Mangin, S. Andrieu, and T. Hauet, Effect of spin transfer torque on domain wall motion regimes in $[\mathrm{Co} / \mathrm{Ni}]$ superlattice wires, Phys. Rev. B 95, 184419 (2017).

[33] F. Gimbert, L. Calmels, and S. Andrieu, Localized electron states \& spin polarization in Co/Ni(111) overlayers, Phys. Rev. B 84, 094432 (2011)

[34] Ia. Lytvynenko, C. Deranlot, S. Andrieu, and T. Hauet, Magnetic tunnel junctions using $\mathrm{Co} / \mathrm{Ni}$ multilayer electrodes with perpendicular magnetic anisotropy, J. Appl. Phys. 117, 053906 (2015).

[35] M. Arora, R. Hübner, D. Suess, B. Heinrich, and E. Girt, Origin or perpendicular magnetic anisotropy in $\mathrm{Co} / \mathrm{Ni}$ multilayers, Phys. Rev. B 96, 024401 (2017).

[36] P. Bruno, Tight-binding approach to the orbital magnetic moment and magnetocrystalline anisotropy of transition-metal monolayers, Phys. Rev. B 39, 865 (1989).

[37] S. Andrieu, L. Calmels, T. Hauet, F. Bonell, P. Le Fevre, and F. Bertran, Spectroscopic \& transport studies of $\mathrm{Co}_{\mathrm{x}} \mathrm{Fe}_{1-\mathrm{x}} / \mathrm{MgO}$ based MTJs, Phys. Rev. B 90, 214406 (2014).

[38] Y. Kamada, H. Kasai, T. Kingetsu, and M. Yamamoto, Structure \& magnetic properties of $\mathrm{Au} / \mathrm{Ni} / \mathrm{Ag} \& \mathrm{Ag} / \mathrm{Ni} / \mathrm{Au}$ superlattices, J. Magn. Soc. Jpn. 23, 581 (1999).

[39] J. Stöhr and H. König, Determination of Spin- and OrbitalMoment Anisotropies in Transition Metals by Angle-Dependent X-Ray Magnetic Circular Dichroism, Phys. Rev. Lett. 75, 3748 (1995).

[40] P. Ohresser, E. Otero, F. Choueikani, K. Chen, S. Stanescu, F. Deschamps, T. Moreno, F. Polack, B. Lagarde, J-P. Daguerre, F. Marteau, F. Scheurer, L. Joly, J.-P. Kappler, B. Muller, O. Bunau, and P. Sainctavit, DEIMOS: A beamline dedicated to dichroism measurements in the 350-2500 eV energy range, Rev. Sci. Instrum. 85, 013106 (2014).

[41] L. Joly, E. Otero, F. Choueikani, F. Marteau, L. Chapuis, and P. Ohresser, Fast continuous energy scan with dynamic coupling of the monochromator and undulator at the DEIMOS beamline, J. Synchrotron Radiat. 21, 502 (2014).

[42] F. Wilhelm, P. Poulopoulos, P. Srivastava, H. Wende, M. Farle, K. Baberschke, M. Angelakeris, N. K. Flevaris, W. Grange, J.-P. Kappler, G. Ghiringhelli, and N. B. Brookes, Magnetic anisotropy energy and the anisotropy of the orbital moment of $\mathrm{Ni}$ in Ni/Pt multilayers, Phys. Rev. B 61, 8647 (2000).

[43] R. Nakajima, J. Stohr, and Y. U. Idzerda, Electron-yield saturation effects in L-edge $\mathrm{X}$-ray magnetic circular dichroism spectra of Fe, Co, and Ni, Phys. Rev. B 59, 6421 (1999).

[44] M. Sicot, S. Andrieu, F. Bertran, and F. Fortuna, Electronic properties of $\mathrm{Fe}, \mathrm{Co}$, and $\mathrm{Mn}$ ultrathin films at the interface with MgO(001), Phys. Rev. B 72, 144414 (2005).

[45] G. van der Laan, Microscopic origin of magneto-crystalline anisotropy in transition metal thin films, J. Phys.: Condens. Matter 10, 3239 (1998).

[46] H. A. Dürr and G. van der Laan, Magnetic circular x-ray dichroism in transverse geometry: Importance of noncollinear ground state moments, Phys. Rev. B 54, R760 (1996).

[47] D. Weller, J. Stohr, R. Nakajima, A. Carl, M. G. Samant, C. Chappert, R. Megy, P. Beauvillain, P. Veillet, and G. A. Held, Microscopic Origin of Magnetic Anisotropy in $\mathrm{Au} / \mathrm{Co} / \mathrm{Au}$ Probed with X-Ray Magnetic Circular Dichroism, Phys. Rev. Lett. 75, 3752 (1995). 
[48] R. Feidenhans'l, Surface structure determination by X-ray diffraction, Surf. Sci. Rep. 10, 105 (1989).

[49] I. K. Robinson, Surface Crystallography, Handbook on Synchrotron Radiation Chap. 7, edited by G. Brown and D. E. Moncton (North-Holland, Amsterdam, 1991), Vol. 3.

[50] G. Renaud, Oxide surfaces and metal/oxide interfaces studied by grazing incidence X-ray scattering, Surf. Sci. Rep. 32, 1 (1998).

[51] E. Vlieg, ROD, a program for surface X-ray crystallography, J. Appl. Crystallogr. 33, 401 (2000).
[52] J. Wan, Y. L. Fan, D. W. Gong, S. G. Shen, and X. Q. Fan, Surface relaxation and stress of fcc metals: $\mathrm{Cu}, \mathrm{Ag}, \mathrm{Au}, \mathrm{Ni}, \mathrm{Pd}, \mathrm{Pt}, \mathrm{Al}$ and Pb, Modell. Simul. Mater. Sci. Eng. 7, 189 (1999).

[53] P. Blaha, K. Schwarz, P. Sorentin, and S. B. Trickey, Fullpotential, linearized augmented plane wave programs for crystalline systems, Comput. Phys. Commun. 59, 399 (1990).

[54] J. P. Perdew, K. Burke, and M. Ernzerhof, Generalized Gradient Approximation Made Simple, Phys. Rev. Lett. 77, 3865 (1996). 